\title{
An analysis of the convergence of Newton iterations for solving elliptic Kepler's equation
}

\author{
A. Elipe · J. I. Montijano · L. Rández • \\ M. Calvo
}

\begin{abstract}
In this note a study of the convergence properties of some starters $E_{0}=$ $E_{0}(e, M)$ in the eccentricity-mean anomaly variables for solving the elliptic Kepler's equation (KE) by Newton's method is presented. By using a Wang Xinghua's theorem (1999) on best possible error bounds in the solution of non-linear equations by Newton's method we obtain for each starter $E_{0}(e, M)$ a set of values $(e, M) \in[0,1) \times[0, \pi]$ that lead to the $q$-convergence in the sense that Newton's sequence $\left(E_{n}\right)_{n \geq 0}$ generated from $E_{0}=E_{0}(e, M)$ is well defined, converges to the exact solution $E^{*}=E^{*}(e, M)$ of $\mathrm{KE}$ and further $\left|E_{n}-E^{*}\right| \leq q^{2^{n}-1}\left|E_{0}-E^{*}\right|$ holds for all $n \geq 0$. This study completes in some sense the results derived by Avendaño et al. (2014) by using Smale's $\alpha$-test with $q=1 / 2$. Also since in KE the convergence rate of Newton's method tends to zero as $e \rightarrow 0$, we show that the error estimates given in the Wang Xinghua's theorem for KE can also be used to determine sets of $q$-convergence with $q=e^{k} \widetilde{q}$ for all $e \in[0,1)$ and a fixed $\widetilde{q} \leq 1$. Some remarks on the use of this theorem to derive a priori estimates of the error $\left|E_{n}-E^{*}\right|$ after $n$ Kepler's iterations are given. Finally, a posteriori bounds of this error that can be used to a dynamical estimation of the error are also obtained.
\end{abstract}

\section{Introduction}

The iterative solution of elliptic Kepler's equation

$$
E-e \sin E=M
$$

where $M$ is the mean anomaly (for periodicity in $[0, \pi]$ ), $e \in[0,1)$ the eccentricity of an elliptic orbit and the unknown $E$ the eccentric anomaly, has been the subject of an extensive list of references for a long time. Here we mention just a few of them (Smith (1979); Broucke (1980); Mikkola (1987); Colwell (1993); Markley (1995); Odell and Gooding (1986); Feinstein and McLaughlin (2006); Charles and Tatum (1998); Palacios (2002); Mortari and Clochiatti (2007); Dubinov and Galidakis (2007); Davis et al. (2010); Calvo et al. (2013); Avendaño et al. (2015); Calvo et al. (2017)).

A. Elipe, J. I. Montijano, L. Rández, M. Calvo

Dpto. Matemática Aplicada - IUMA. Universidad de Zaragoza, 50009 Zaragoza, Spain

A. Elipe

Centro Universitario de la Defensa, 50090 Zaragoza. Spain 
In many cases, taking a suitable starter $E_{0}=E_{0}(e, M)$, Newton's method is applied to the equation $f_{e}(E ; M) \equiv E-e \sin E-M=0$ with the recursion

$$
E_{k+1}=N\left(E_{k}\right) \equiv E_{k}-\frac{f_{e}\left(E_{k} ; M\right)}{f_{e}^{\prime}\left(E_{k} ; M\right)}, \quad k=0,1, \ldots
$$

where $N(\cdot)=N_{f_{e}}(\cdot)$ is the Newton's iterator of $f_{e}$, to get a Newton's sequence $\left(E_{k}\right)_{k \geq 0}$ that converges to the unique solution $E^{*}=E^{*}(e, M)$ of $f_{e}(E ; M)=0$. Then our aim is to make the small number $n$ of iterations so that $E_{n}$ provides an approximation to $E^{*}$ with the desired accuracy. Observe that for $e=1$ and $E_{k}=0$ the recursion (2) becomes singular and therefore we expect to find some difficulties with the convergence when $(e, M)$ is close to $(1,0)$.

First of all observe that the Newton's iterator $N\left(E_{k}\right)$ defined in (2) is well defined for all $M \in[0, \pi]$ and $e \in[0,1)$ for any $E_{k}$, then for all starter $E_{0}=E_{0}(e, M)$ the corresponding sequence $\left(E_{n}\right)_{n \geq 0}$ is well defined. However we cannot ensure their convergence to some $E^{*}$ that would be the unique solution $E^{*}=E^{*}(e, M)$ of $f_{e}(E ; M)=0$. In fact it has been shown in Charles and Tatum (1998) that with the standard choice $E_{0}(e, M)=M$, while there is convergence for $(e, M)=(0.991,0.13 \pi)$ and for $(e, M)=$ $(0.993,0.13 \pi)$, Newton's method seems not converge for $(e, M)=(0.992,0.13 \pi)$. This implies that for a given starter $E_{0}(e, M)$ it is important to identify the set of $(e, M) \in$ $[0,1) \times[0, \pi]$ that ensures the convergence of Newton's iterates.

There are some particular starters that lead to convergent Newton's sequences. Thus if $0 \leq f_{e}\left(E_{0}(e, M)\right) \leq \pi$, taking into the monotonic increasing and convexity of $f_{e}(E ; M)$ with respect to $E$ with respect to $E \in[0, \pi]$ for all $M \in[0, \pi]$ and $e \in[0,1)$ we have $f_{e}\left(E^{*}(e, M) ; M\right)=0 \leq f_{e}\left(E_{0}(e, M) ; M\right)$ which by monotony of $f_{e}$ implies $E^{*}(e, M) \leq E_{0}(e, M)$. Now by the convexity of $f_{e}$ the sequence $\left(E_{k}\right)_{k>0}$ decreases monotoniquely to $E^{*}$. Therefore $0 \leq f_{e}\left(E_{0}(e, M)\right) \leq \pi$ is a sufficient condition to ensure the convergence of Newton's sequence. In particular for the simplest guess $E_{0}=$ $E_{0}(e, M)=\pi$ we have the desired monotone decreasing convergence.

A second point concerns the rate of convergence of a convergent Newton's sequence $\left(E_{k}\right)_{k \geq 0}$ starting from $E_{0}=E_{0}(e, M)$ as a function of $(e, M) \in[0,1) \times[0, \pi]$. Of course Newton's method is a second order method and we have

$$
\lim _{k \rightarrow \infty} \frac{\left|E_{k+1}-E^{*}\right|}{\left|E_{k}-E^{*}\right|^{2}}=\frac{e\left|\sin \left(E^{*}\right)\right|}{2\left(1-e \cos \left(E^{*}\right)\right)},
$$

and this shows that the so called quotient convergence factor (Ortega and Rheinboldt (1970), pp. 282) given by the right hand side of (3) may become arbitrary large for $\left(e, E^{*}\right)$ close to $(1,0)$ i.e. the singular point of the Newton's iteration. Moreover this quadratic convergence only holds for $E_{k}$ in a sufficiently small neighborhood of $E^{*}$ that is not known in advance.

In general to study the convergence of Newton iterations starting from $x_{0}$ for solving a non linear system $F(x)=0$ with exact solution $x^{*}$, there are several results depending on the assumptions of $F$ around $x_{0}$. Some well known are the NewtonKantorovitch's (Kantorovich and Akilov (1982)) and Smale's (Smale (1986)) theorems revised more recently in (Dedieu (2006); Argyros et al. (2014)). These results depend on the measure of the error in the approximation to the exact solution $x^{*}$. Some frequently used measures are

$$
e d\left(x_{n}\right)=\left\|x_{n+1}-x_{n}\right\|,
$$


that appears e.g. in the original paper of Smale (1986) and

$$
e g\left(x_{n}\right)=\left\|x^{*}-x_{n}\right\| \quad e s\left(x_{n}\right)=\left\|F^{\prime}\left(x_{0}\right)^{-1} F\left(x_{n}\right)\right\|,
$$

that are two natural measures: the first one in the sense of the global error and the second one considered by Smale in Blum et al. (2010).

Here for a starter $E_{0}=E_{0}(e, M)$ of $\operatorname{KE~} f_{e}(E ; M) \equiv E-e \sin E-M=0$ well defined for all $(e, M) \in[0,1) \times[0, \pi]$ we introduce the following

Definition 1 The starter $E_{0}$ of $\mathrm{KE}$ is $q$-convergent for $(e, M) \in[0,1) \times[0, \pi]$ if the Newton's sequence $\left(E_{n}\right)_{n \geq 0}$ defined by (2) converges to some $E^{*}$, the unique solution of $f_{e}(E ; M)=0$, and there exist some $q \in[0,1)$ such that the following estimate holds

$$
\left|E_{n}-E^{*}\right| \leq q^{2^{n}-1}\left|E_{0}-E^{*}\right| \quad n=0,1,2, \ldots
$$

Moreover for a given $q \in[0,1)$ the set of $q$-convergent values $(e, M) \in[0,1) \times[0, \pi]$ will be called the $q$-convergence set of the starter $E_{0}=E_{0}(e, M)$.

Note that for $q=1 / 2, E_{0}$ is an approximate zero of KE in Smale's notation (Smale (1986), Avendaño et al. (2014)).

The aim of this paper is to use a version of Newton-Kantorovich theorem given by Wang Xinghua (1999) that obtains optimal error estimates of Newton's sequence to derive sufficient conditions of $q$-convergence of a starter $E_{0}=E_{0}(e, M)$ for $\mathrm{KE}$ and the corresponding $q$-convergence set. Note a similar study with $q=1 / 2$ has been carried out in Avendaño et al. (2014) by using the so called $\alpha$-test based on Smale's theorem Blum et al. (2010). Here it will be seen that for many starters the $q$-factor can be written in the form $q=e^{k} \widetilde{q}$ with some $0 \leq \widetilde{q}<1$ and $k \geq 0$ which, in view of (6), imply sharper error bounds for small eccentricities whereas in Avendaño et al. (2014) the bound is uniform in the eccentricity. Further, other estimates (4)-(5) are derived that allow us a priori and a posterior estimates of the error after $n$ iterations. The paper is organized as follows: In Section 2 the original Wang Xinghua version of of Newton-Kantorovich theorem as well their particular case for Kepler's equation are presented. In Section 3 the $q$-convergence properties of some well known starters are studied including other error estimates. The paper ends with some conclusions on the use of these results in practical applications.

\section{Error estimates in Newton's iteration of Kepler equation}

First of all we recall the version of Newton-Kantorovich theorem given by Wang Xinghua (1999) in Th. 4.3.

Theorem 1 Let $X$ and $Y$ be Banach spaces, $D$ an open convex subset of $X$ and $F: D \subset X \rightarrow Y$ be a Frechet differentiable non linear operator. Assume that $F^{\prime}\left(x_{0}\right)$ is invertible for a given starting point $x_{0} \in D$ and that there exist positive constants $\beta, L$ and $\lambda$ so that

1.

$$
\beta=\left\|F^{\prime}\left(x_{0}\right)^{-1} F\left(x_{0}\right)\right\|
$$

2.

$$
\left\|F^{\prime}\left(x_{0}\right)^{-1}\left(F^{\prime}(y)-F^{\prime}(z)\right)\right\| \leq L\|y-z\| \quad \text { for all } y, z \in D
$$


3.

$$
\lambda=L \beta<1 / 2 .
$$

4. The closed ball of center $x_{0}$ and radius $r=(1-\sqrt{1-2 \lambda}) / L, B\left(x_{0}, r\right)=\{x ; \| x-$ $\left.x_{0} \| \leq r\right\}$ satisfies $B\left(x_{0}, r\right) \subset D$.

Then $F^{\prime}(x)$ is invertible in $B\left(x_{0}, r\right)$ and the sequence $\left(x_{k}\right)_{k \geq 0}$ given by

$$
x_{k+1}=x_{k}-F\left(x_{k}\right)^{-1} F\left(x_{k}\right), \quad k=0,1, \ldots
$$

remains in $B\left(x_{0}, r\right)$ and converges to some $x^{*} \in B\left(x_{0}, r\right)$ with $F\left(x^{*}\right)=0$ and the following (optimal) estimates hold

$$
e g\left(x_{n}\right) \leq \frac{q^{2^{n}-1}}{\mu_{n}(q)} e g\left(x_{0}\right) \leq \frac{q^{2^{n}-1}}{\mu_{n-1}\left(q^{2}\right)} e d\left(x_{0}\right)
$$

and

$$
\frac{e d\left(x_{n}\right)}{\xi\left(q^{2^{n}}\right)} \leq e g\left(x_{n}\right) \leq \frac{q}{\beta} \mu_{n-1}\left(q^{2}\right) e d\left(x_{n-1}\right)^{2} \leq q^{2^{n}-1} e d\left(x_{n-1}\right)
$$

where $q$, and the functions $\mu_{n}$ and $\xi$ are given by

$$
\begin{gathered}
q=q(\lambda)=\frac{1-\sqrt{1-2 \lambda}}{1+\sqrt{1-2 \lambda}}=\frac{2 \lambda}{(1+\sqrt{1-2 \lambda})^{2}}, \quad \lambda=\frac{2 q}{(1+q)^{2}} . \\
\mu_{n}(\omega)=\sum_{i=0}^{2^{n}-1} \omega^{i}=\frac{1-\omega^{2^{n}}}{1-\omega} \geq 1 \quad(0 \leq \omega<1) \\
\xi(\omega)=\frac{1+\sqrt{1+4 \omega /(1+\omega)^{2}}}{2} .
\end{gathered}
$$

Note that according to Eq. (12) when $\lambda \in(0,1 / 2)$ the corresponding $q \in(0,1)$. Hence to have $q=1 / 2$ we must take $\lambda=4 / 9$ and for $q=1 / 10$ we must take $\lambda=20 / 121$. Further observe that for $\omega \in[0,1]$ the function $\xi(\omega)$ is monotonic increasing and therefore

$$
1=\xi(0)<\xi(\omega)<\xi(1)=\frac{1+\sqrt{2}}{2}, \quad \text { for } \quad 0<\omega<1 .
$$

In the case of $\mathrm{KE}, D=X=Y=\mathbb{R}, x=E$ and

$$
F(x)=f_{e}(E ; M) \equiv x-e \sin x-M, \quad \text { with } e \in[0,1), M \in[0, \pi]
$$

is analytic for all $x \in \mathbb{R}$.

Denoting the starting value for the solution of (14) by $x_{0}=E_{0}=E_{0}(e, M)$ the constants $\beta$ and $L$ of (7), (8) are

$$
\beta=\frac{\left|E_{0}-e \sin E_{0}-M\right|}{1-e \cos E_{0}}, \quad L=\frac{e}{1-e \cos E_{0}},
$$

and therefore for each $E_{0}, \lambda=\lambda\left(E_{0}\right)$ is a function of $e$ and $M$ defined by (9) and given by

$$
\lambda=\lambda\left(E_{0}\right)=\frac{e\left|E_{0}-e \sin E_{0}-M\right|}{\left(1-e \cos E_{0}\right)^{2}}=\frac{e\left|E_{0}(e, M)-e \sin \left(E_{0}(e, M)\right)-M\right|}{\left(1-e \cos \left(E_{0}(e, M)\right)\right)^{2}} .
$$

Hence applying Theorem (1) to the KE: $f_{e}(E ; M)=0$ we have 
Proposition 1 Let $E_{0}=E_{0}(e, M)$, be a given starter for solving $K E$, then for all $\nu \in(0,1 / 2)$ the set

$$
\Sigma_{q}\left(E_{0}\right)=\left\{(e, M) \in[0,1) \times[0, \pi] ; \lambda\left(E_{0}\right) \leq \nu<1 / 2\right\}
$$

with $q=q(\nu)$ given by (12) is contained in the $q$-convergence set of $E_{0}$. In addition the following estimates hold

$$
\begin{gathered}
\left|E^{*}-E_{n}\right| \leq \frac{q^{2^{n}-1}}{\mu_{n}(q)}\left|E^{*}-E_{0}\right| \leq q^{2^{n}-1}\left|E^{*}-E_{0}\right| \\
\left|E^{*}-E_{n}\right| \leq \frac{q^{2^{n}-1}}{\mu_{n-1}\left(q^{2}\right)}\left|E_{1}-E_{0}\right| \leq q^{2^{n}-1}\left|E_{1}-E_{0}\right|, \\
\left|E^{*}-E_{n}\right| \leq q^{2^{n}-1}\left|E_{n}-E_{n-1}\right|, \\
\left|E_{n+1}-E_{n}\right| \leq \xi\left(q^{2^{n}}\right)\left|E^{*}-E_{n}\right| \leq\left(\frac{1+\sqrt{2}}{2}\right)\left|E^{*}-E_{n}\right| .
\end{gathered}
$$

Next we will derive some consequences that follow from the above Proposition: First of all for a given starter $E_{0}$ the set $\Sigma_{q}\left(E_{0}\right)$ is contained in the $q$-convergence set of $E_{0}$ with rate $q=q(\nu)<1$ gives a useful information about the values of eccentricity and mean anomaly that satisfy the above bounds (18) to (21) and therefore on the $q$ convergence with this factor. Clearly starters with larger sets $\Sigma_{q}\left(E_{0}\right)$ or smaller values of $q$ will be preferred for Newton's solution of KE.

Secondly in view of (16) there is a $k \geq 1$ such that

$$
\lambda\left(E_{0}\right)=e^{k} \lambda_{k}\left(E_{0}\right), \quad \text { with }\left.\quad \lambda_{k}\left(E_{0}\right)\right|_{e \rightarrow 0} \neq 0 .
$$

Then $q(\lambda)$, for $\lambda \in[0,1 / 2]$ given by (12) can be written as

$$
\begin{aligned}
q(\lambda) & =\frac{2 \lambda}{(1+\sqrt{1-2 \lambda})^{2}}=e^{k} \frac{2 \lambda_{k}}{\left(1+\sqrt{1-2 e^{k} \lambda_{k}}\right)^{2}} \\
& \leq e^{k} \frac{2 \lambda_{k}}{\left(1+\sqrt{1-2 \lambda_{k}}\right)^{2}}=e^{k} q\left(\lambda_{k}\right) .
\end{aligned}
$$

Thus for $0<\rho<1$ we may define the sets

$$
\widetilde{\Sigma}_{\rho}\left(E_{0}\right)=\left\{(e, M) \in[0,1) \times[0, \pi] ; q\left(\lambda_{k}\right) \leq \rho<1\right\},
$$

so that for the starter $E_{0}=E_{0}(e, M)$ if $(e, M) \in \widetilde{\Sigma}_{\rho}\left(E_{0}\right)$ we have $q$-convergence with the factor $q=e^{k} \rho$ for all $e \in[0,1)$ and now the $q$-convergence factor tends to zero as $e^{k}$, in contrast with the information derived from the sets $\Sigma_{q}\left(E_{0}\right)$ in which the convergence is uniform in the whole set.

Further observe that the inequality (18) bounds the global error in the $n$-th iteration $e g\left(E_{n}\right)=\left|E^{*}-E_{n}\right|$ in terms of the global error in the starting value $e g\left(E_{0}\right)=\left|E^{*}-E_{0}\right| \leq \pi$. Clearly the factor $q^{2^{n}-1}$ with $0<q<1$ implies the desired $q$-convergence and it can be considered as an a priori error bound after a given number $n$ of iterations. Also if we want $\left|E^{*}-E_{n}\right| \leq E$ tol it is enough to select $n>0$ 
so that $\delta_{n}=\delta_{n}(e, M)=q^{2^{n}-1}\left|E_{1}-E_{0}\right| \leq$ Etol. For example, since $\left|E^{*}-E_{0}\right| \leq \pi$ for all $E_{0}$, if $(e ; M) \in \Sigma_{1 / 10}\left(E_{0}\right)$, after four Kepler's iterations $(n=4)$ we can ensure a global error $\left|E^{*}-E_{4}\right| \leq \pi \times 10^{-15}$.

The inequality (20) provide an additional information with a more practical value because $\left|E_{n}-E_{n-1}\right|$ is available along the iterative process and then allow us to choose dynamically the number of iterations depending on our accuracy requirements. Thus if we want $\left|E^{*}-E_{n}\right| \leq E$ tol it is enough to check $q^{2^{n}-1}\left|E_{n}-E_{n-1}\right| \leq$ Etol. Finally, (21) provides a two-sided error bound of the global error $\left|E^{*}-E_{n}\right|$.

\section{The convergence of some starters}

Next we examine the convergence properties of some well known starters in the literature $E_{0}=E_{0}(e ; M)$ obtaining the sets $\Sigma_{q}\left(S_{0}\right) \subset[0,1) \times[0, \pi]$ that ensure the convergence bounds (18)-(21) for $\nu \leq 1 / 2$. We have included the value $\nu=1 / 2$ corresponding to $q(1 / 2)=1$ because this is the boundary of $q$-convergence ensured by Proposition 1. In particular we consider the values of $\nu=1 / 2,4 / 9,8 / 25,20 / 121$ that correspond to the $q$-factors $q=1,1 / 2,1 / 4,1 / 10$ respectively. Note that $q=1 / 2$ is the corresponding to Smale $\alpha$-test. For some starters we have used the notations $S_{j}$ employed by Odell and Gooding (1986) in Table I.

\subsection{The starter $E_{0}=\pi$.}

This is one of the simplest starters considered in the literature and by (2) the first Newton's iteration gives $N_{f_{e}}(\pi)=(M+\pi e) /(1+e) \in[0, \pi]$ that has a low computational cost (does not require the computation of trigonometric functions or roots). Because of this we will study this starter from the first iteration i.e.

$$
E_{0}=E_{0}(e ; M)=\frac{M+\pi e}{1+e},
$$

instead of the above $E_{0}=\pi$. Now the function $\lambda\left(E_{0}\right)$ of (16) becomes

$$
\lambda\left(E_{0}\right)=\lambda\left(E_{0}\right)(e, M)=\frac{e^{2}[\pi-(1+e) \sin ((M+\pi e) /(1+e))-M]}{(1+e)\left(1-e \cos ((M+\pi e) /(1+e))^{2}\right.},
$$

and for $\nu<1 / 2$ the set $\Sigma_{q}\left(E_{0}\right)$ is defined implicitely by

$$
\Sigma_{q}\left(E_{0}\right)=\left\{(e, M) \in[0,1) \times[0, \pi] ; \lambda\left(E_{0}\right)(e, M) \leq \nu<1 / 2\right\},
$$

where $q=q(\nu)$ is given by (12).

In Figure 1 we display the boundaries of the sets $\Sigma_{q}\left(E_{0}\right)$ for $\nu=1 / 2,4 / 9,8 / 25,20 / 121$ corresponding to the $q$-factors $q=1,1 / 2,1 / 4,1 / 10$. Obviously $q_{1}<q_{2} \leq 1$ implies that $\Sigma_{q_{1}}\left(E_{0}\right) \subset \Sigma_{q_{2}}\left(E_{0}\right)$. Here the sets $\Sigma_{q}\left(E_{0}\right)$ have the points $(e, M) \in[0,1) \times[0, \pi]$ above the corresponding boundaries.

From the Proposition 1 it follows the $q$-convergence of starter (25) with factor $q<1$ for all $(e, M)$ above the boundary of $\Sigma_{1}\left(E_{0}\right)$. In particular for all $e<0.461359=e_{1}$ this property holds for all $M$ and this implies that solving KE along an orbit with eccentricity $e<e_{1}$ we have $q$-convergence for all values of the mean anomaly. Here $e_{1}$ is the unique root of $\lambda\left(E_{0}\right)(e, M=0)=1 / 2$ in $(0,1)$. Similar conclusions follow 
from the boundaries of the the other sets $\Sigma_{q}\left(E_{0}\right)$. Thus, in the case of $q=1 / 2$ for $e<0.42019$ (the root of $\left.\lambda\left(E_{0}\right)(e, M=0)=4 / 9\right)$ we have $q$-convergence with factor $q \leq 1 / 2$. On the other hand for $M>0.155763$ we have $q$-convergence with factor $q<1$ for all values of eccentricity.

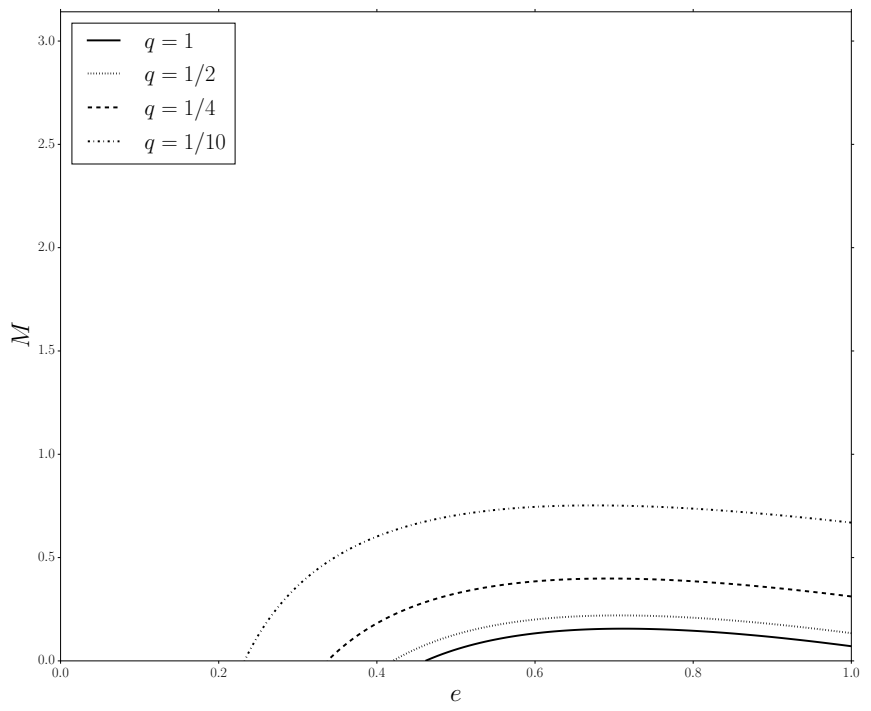

Fig. 1 Boundaries of the $\Sigma_{q}\left(E_{0}\right)$ sets of starter (25) for the values of factor $q=$ $1,1 / 2,1 / 4,1 / 10$. The sets $\Sigma_{q}$ are the points $(e, M) \in[0,1) \times[0, \pi]$ above the corresponding boundaries.

Some relevant consequences for small eccentricities can be derived from the scaled $q$-convergence sets

$$
\widetilde{\Sigma}_{\rho}\left(E_{0}\right)=\left\{(e, M) \in[0,1) \times[0, \pi] ; \widetilde{q}\left(\lambda_{2}\left(E_{0}\right)\right) \leq \rho \leq 1\right\}
$$

Here

$$
\lambda_{2}\left(E_{0}\right)=\lambda_{2}\left(E_{0}\right)(e, M)=\frac{[\pi-(1+e) \sin ((M+\pi e) /(1+e))-M]}{(1+e)\left(1-e \cos ((M+\pi e) /(1+e))^{2}\right.} .
$$

In Figure 2 we display the boundaries of the scaled $\rho$-convergence sets for $\rho=1,1 / 2,1 / 4,1 / 10$. These scaled sets include the points of $\widetilde{\Sigma}_{\rho}\left(E_{0}\right)$ i.e $(e, M) \in[0,1) \times[0, \pi]$ above the corresponding boundaries in which $q=e^{2} \rho$. From this Figure 2 it follows that e.g. in the case of $\rho=1 / 2$ for $M>1.2$ we have $q$-convergence with factor $q<(1 / 2) e^{2}$.

To illustrate the upper bound (20) of the error $\left|E^{*}-E_{n}\right|$ in the $n$-th Newton's iteration as a function of the last two iterations in a region of $q$-convergence we consider the solution of an elliptic orbit with eccentricity $e=0.3$. In Figure 3 we display for $M \in[0, \pi]$ the values of $E x$ of the upper bound $q^{2^{n}-1}\left|E_{n}-E_{n-1}\right|=10^{-E x}$ for 


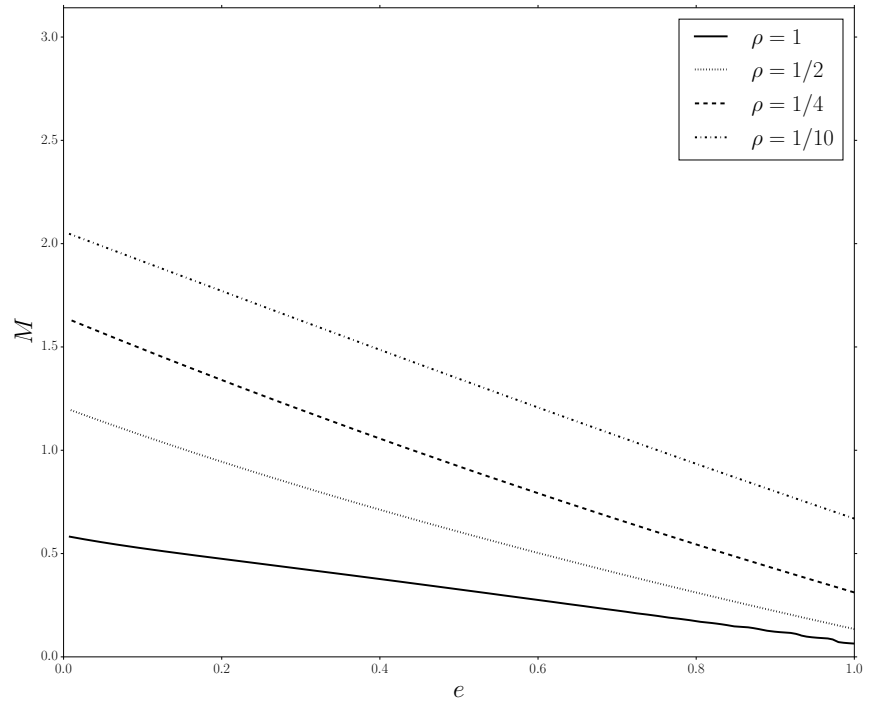

Fig. 2 Boundaries of the scaled sets $\widetilde{\Sigma}_{\rho}$ of starter (25) for the values $\rho=1,1 / 2,1 / 4,1 / 10$. The $\rho$-convergence scaled sets $\widetilde{\Sigma}_{\rho}$ are the points $(e, M) \in[0,1) \times[0, \pi]$ above the corresponding boundaries in which $q=e^{2} \rho$.

eccentricity $e=0.3$ and $n=2,3,4,5$ (Here $E x$ is limited between 0 and 60). Observe that for eccentricity $e=0.3$ all values of $M$ are included in the $q$-convergence region $\Sigma_{1 / 4}\left(S_{0}\right)$ therefore we take $q=1 / 4$ in the above upper bound.

Finally, in Fig. 4, we display the number of correct figures in the error after $n=$ 1, 2, 3, 4 iterations of Newton's method. The quadratic convergence of Newton's method can be appreciated because from an iteration to the next one it can be noted that approximately the same level curve duplicates their value. These figures show the regions in $(e, M) \in[0,1) \times[0, \pi]$ with a number of correct digits in the error $\left|E^{*}-E_{n}\right|$ after $n$ iterations.

3.2 The starter $E_{0}=0$.

Now the first Newton's iteration gives $N_{f_{e}}(0)=M /(1-e)$ that has a low computational cost. Then, as in the previous starter, we will consider instead of $E_{0}=0$ the starter after the first iteration given by

$$
E_{0}=\min \{M /(1-e), \pi\}=\left\{\begin{array}{l}
M /(1-e) \quad \text { for } M<\pi(1-e) \\
\pi \quad \text { otherwise }
\end{array}\right.
$$

and the $q$-convergence regions are defined by (17)

In Figure 5 we display the boundaries of the $q$-convergence regions $\Sigma_{q}\left(E_{0}\right)$ for $\nu=1 / 2,4 / 9,8 / 25,20 / 121$ corresponding to the $q$-factors $q=1,1 / 2,1 / 4,1 / 10$. Here 


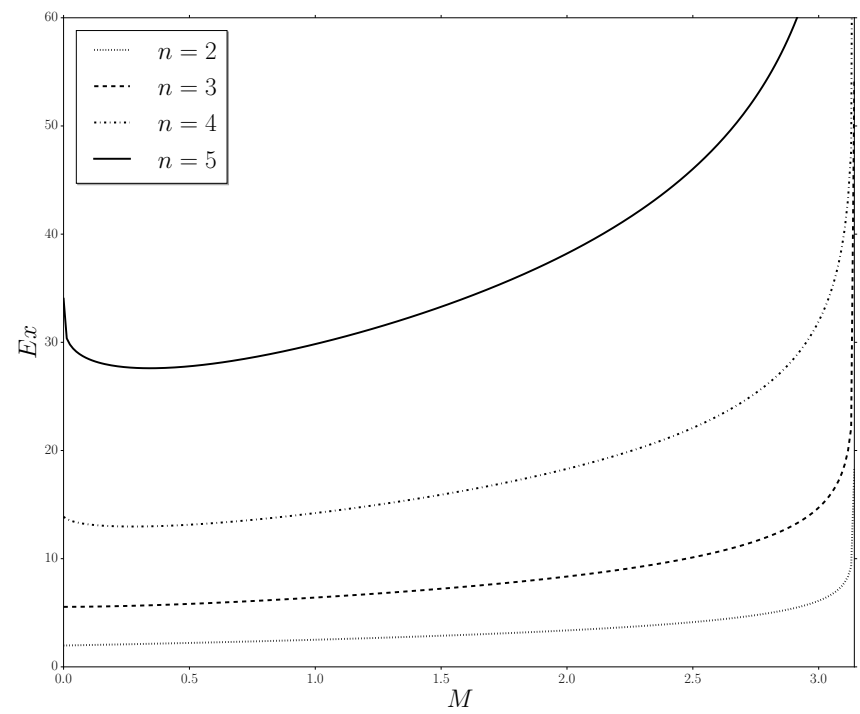

Fig. 3 Bounds Etol $=10^{-E x}$ of the errors $\left|E^{*}-E_{n}\right|$ given by Prop. 1 for $e=0.3$ and $n=2,3,4,5$

the convergence regions are the sets of $(e, M) \in[0,1) \times[0, \pi]$ outside the corresponding boundaries. A study of scaled $q$-convergence sets and error bounds as in the previous starter is skipped for brevity.

3.3 The starter $S_{1}: E_{0}=M$.

Now the function $\lambda\left(S_{1}\right)$ of (17) becomes

$$
\lambda\left(S_{1}\right)=\frac{e^{2} \sin M}{(1-e \cos M)^{2}},
$$

and the sets of $q$-convergence derived from Proposition 1 can be defined explicitly as a function of $\nu<1 / 2$ by

$$
\Sigma_{q}\left(S_{1}\right)=\{(e, M) ; 0 \leq e \leq \min \{1, \sqrt{\nu} /(\sqrt{\sin M}+\sqrt{\nu} \cos M)\}, \quad M \in[0, \pi]\} .
$$

In Figure 6 we display the boundaries of the sets $\Sigma_{q}\left(E_{0,1}\right)$ for $\nu=1 / 2,4 / 9,8 / 25,20 / 121$ corresponding to the $q$-factors $q=1,1 / 2,1 / 4,1 / 10$. Here the convergence regions are the sets of $(e, M) \in[0,1) \times[0, \pi]$ between the corresponding two boundaries.

Note that as remarked in Odell and Gooding (1986), for $e>0.9733$ there is a range of values of $M$ for which Newton's iterations diverge. 

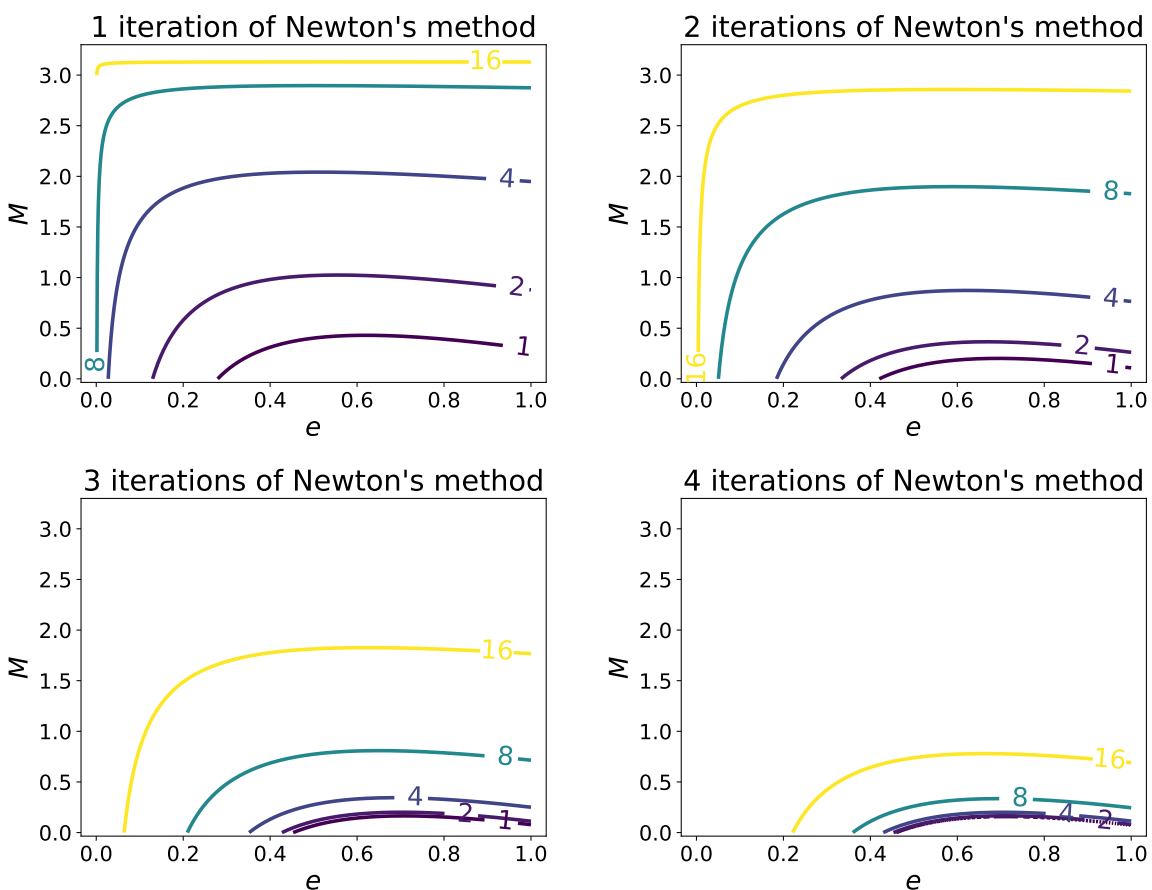

Fig. 4 Level curves indicate the positive decimal logarithm of the error bound $q^{2^{n}-1}\left|E_{1}-E_{0}\right|$ of $\left|E^{*}-E_{n}\right|$ for the starter (25) for $n=2, \ldots, 4$ iterations of Newton's method. Here $q$ is defined by (12) with $\lambda$ given by (16).

3.4 The starter $S_{2}: E_{0}=M+e \sin M$.

Now the function $\lambda\left(S_{2}\right)$ of (17) becomes

$$
\lambda\left(S_{2}\right)=\frac{e^{2}\left|\sin M-\sin E_{0}\right|}{\left(1-e \cos E_{0}\right)^{2}},
$$

and for $\nu<1 / 2$ the set $\Sigma_{q}\left(E_{0}\right)$ is defined implicitely by

$$
\Sigma_{q}\left(S_{2}\right)=\left\{(e, M) \in[0,1) \times[0, \pi] \mid \frac{e^{2}\left|\sin M-\sin E_{0}\right|}{\left(1-e \cos E_{0}\right)^{2}} \leq \nu\right\} .
$$

with $E_{0}=M+e \sin M$. In Figure 7 we display the boundaries of the sets of (29) for $q=1,1 / 2,1 / 4,1 / 10$.

3.5 The starter $S_{4}: E_{0}=M+e$.

Now the function $\lambda\left(S_{4}\right)$ of (17) becomes

$$
\lambda\left(S_{4}\right)=\frac{e^{2}(1-\sin (M+e))}{(1-e \cos (M+e))^{2}},
$$




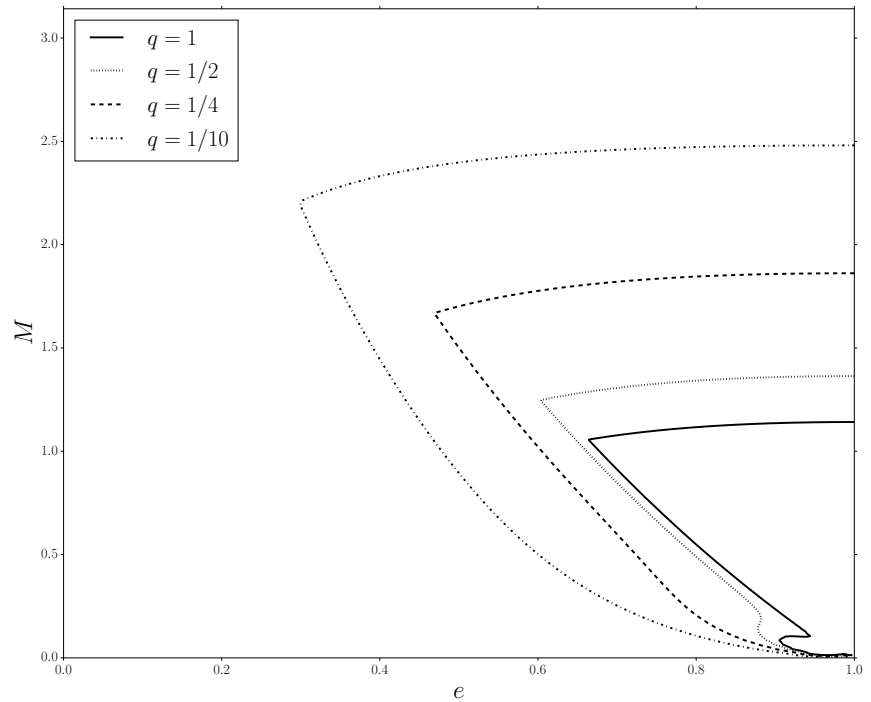

Fig. 5 Boundaries of the $\Sigma_{q}\left(E_{0}\right)$ sets of starter (27) for $q=1,1 / 2,1 / 4,1 / 10$. The sets $\Sigma_{q}$ are the points of $(e, M) \in[0,1) \times[0, \pi]$ on the left of the corresponding boundaries.

and the set $\Sigma_{q}$ is defined implicitly by

$$
\Sigma_{q}\left(S_{4}\right)=\left\{(e, M) \in[0,1) \times[0, \pi] \mid \frac{e^{2}(1-\sin (M+e))}{(1-e \cos (M+e))^{2}} \leq \nu<1 / 2\right\} .
$$

In Figure 8 we display the boundaries of the $q$-convergence sets (30) for $q=1,1 / 2,1 / 4,1 / 10$.

3.6 The starter $S_{7}$

Here the function $E_{0}=E_{0}(e, M)$ is given by

$$
E_{0}(e, M)=\operatorname{Min}\left\{\frac{M}{1-e}, \quad M+e, \frac{M+e \pi}{1+e}\right\}
$$

and it is a piecewise linear function of the mean anomaly.

Taking into account that $\pi \geq E_{0}(e, M) \geq E^{*}(e, M)$ the Newton's sequence starting from $E_{0}$ is monotoniquely decreasing and convergent for all $(e, M) \in[0,1) \times[0, \pi]$.

Concerning the rate of convergence, in Figure 9 we display the boundaries of the $q$-convergence sets $\Sigma_{q}\left(S_{7}\right)$ for $q=1,1 / 2,1 / 4,1 / 10$. Here the corresponding sets are limited between the upper and lower lines. 


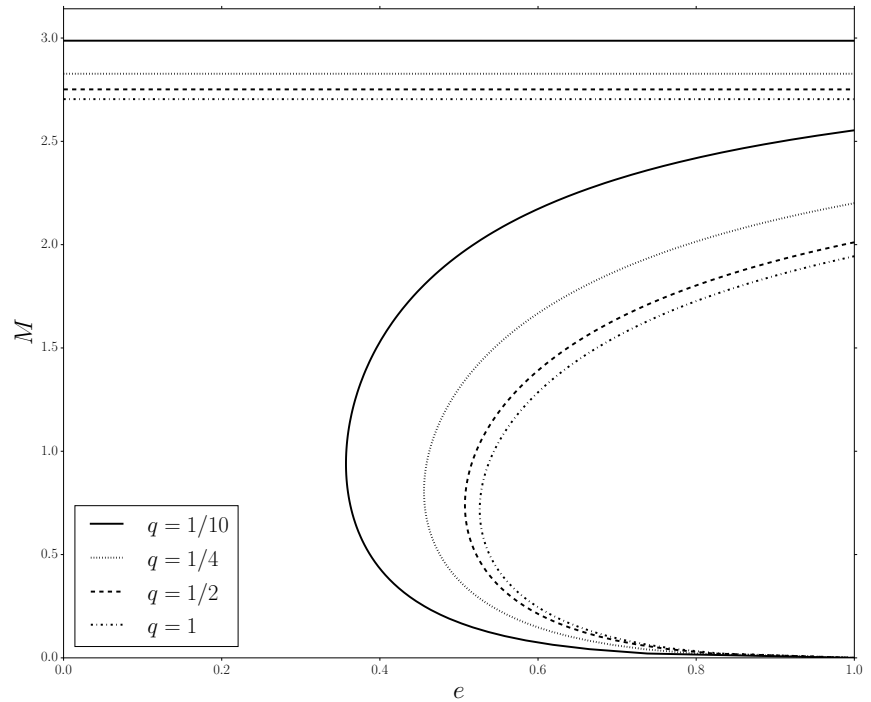

Fig. 6 Boundaries of the $\Sigma_{q}\left(E_{0}\right)$ sets for several values of $q$ of starter $E_{0}=M$.

3.7 The starter of Charles and Tatum (1998)

This starter is given by

$$
E_{0}=E_{0}(e, M)=M+e\left(\left(\pi^{2} M\right)^{1 / 3}-\left(\frac{\pi}{15}\right) \sin M-M\right) .
$$

It is an empirical starter that has been obtained by means of some systematic trial and error and correction by graphical means. The authors claim that in the range $e \in[0.991,1]$ and $M \in[0.001,0.1]$ convergence to nine decimal places was achieved in four Newton's iterations. In Figure 10 we display the $\Sigma_{q}\left(E_{0}\right)$ sets corresponding to the values $\nu=4 / 9(q=1 / 2)$ and $\nu=20 / 121$ in which $q=1 / 10$.

\subsection{Broucke's starter (1980)}

This is a piecewise linear function in the mean anomaly $M$ with coefficients depending on the eccentricity that is given by

$$
E_{0}(e, M)=\left\{\begin{array}{lll}
\gamma(-e) M, & \text { for } & M \in[0,1-e), \\
\xi(-e) M+(e / 2), & \text { for } & M \in[1-e, \pi / 2-e), \\
\gamma(e) M+\nu_{1}(e), & \text { for } & M \in[\pi / 2-e, \pi-e-1), \\
\xi(e) M+\nu_{2}(e), & \text { for } & M \in[\pi-e-1, \pi],
\end{array}\right.
$$




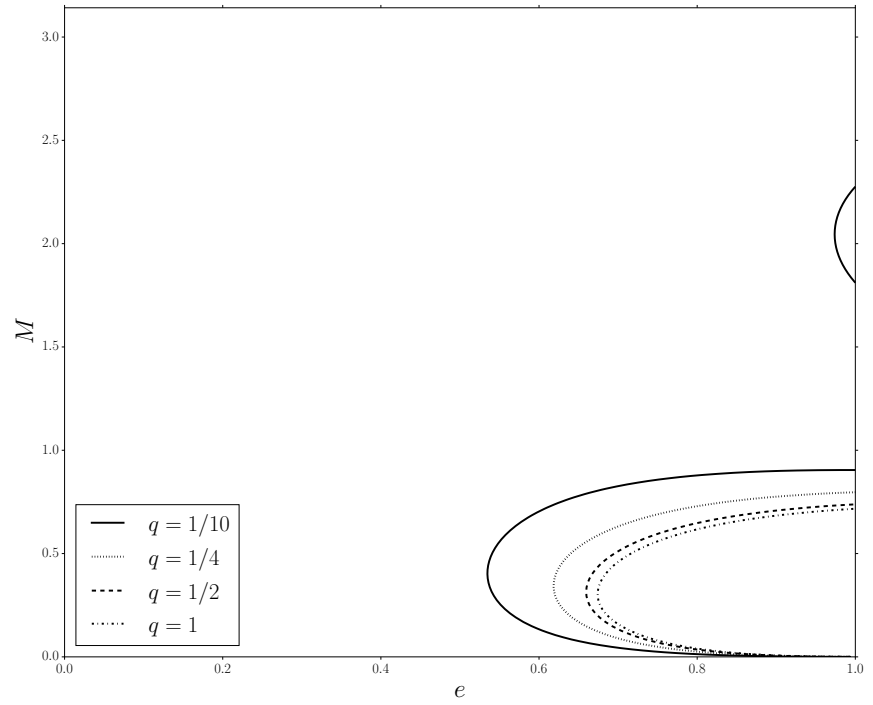

Fig. $7 \Sigma_{q}\left(E_{0}\right)$ sets of starter $S_{2}: E_{0}=M+e \sin M$ for $q=1,1 / 2,1 / 4,1 / 10$. Here the sets $\Sigma_{q}$ include the points $(e, M) \in[0,1) \times[0, \pi]$ under the upper boundary and on the left of the lower boundary.

with

$$
\begin{array}{lll}
\gamma(e)=\frac{2 \pi+(2+\pi) e}{(e+1)(4 e+2 \pi)}, & \xi(e)=\frac{\pi+e}{\pi+2 e} \\
\nu_{1}(e)=\frac{e(3 \pi+2 e)}{2 \pi+4 e}, & \nu_{2}(e)=\frac{e \pi(2+4 e+\pi)}{(e+1)(4 e+4 e+2 \pi)} .
\end{array}
$$

As it has been shown in our paper Calvo et al. (2013), this is globally a good starter in the sense of the $\|\cdot\|_{2}$ and $\|\cdot\|_{1}$ norms of the error in $e \in[0,1)$, and $M \in[0, \pi]$.

In Figure 11 we display the $q$-convergence sets for $q=1,1 / 2,1 / 4,1 / 10$.

\subsection{The starter of Calvo et al. (2013).}

The authors of the present paper have derived an starter that minimizes in a global sense the error for $e \in[0,1), M \in[0, \pi]$. This starter is a piecewise linear function in the mean anomaly given by

$$
E_{0}(e, M)= \begin{cases}p_{1} M+q_{1}, & M \in\left[M_{0}, M_{1}\right], \\ p_{2} M+q_{2}, & M \in\left[M_{1}, M_{2}\right], \\ p_{3} M+q_{3}, & M \in\left[M_{2}, M_{3}\right],\end{cases}
$$




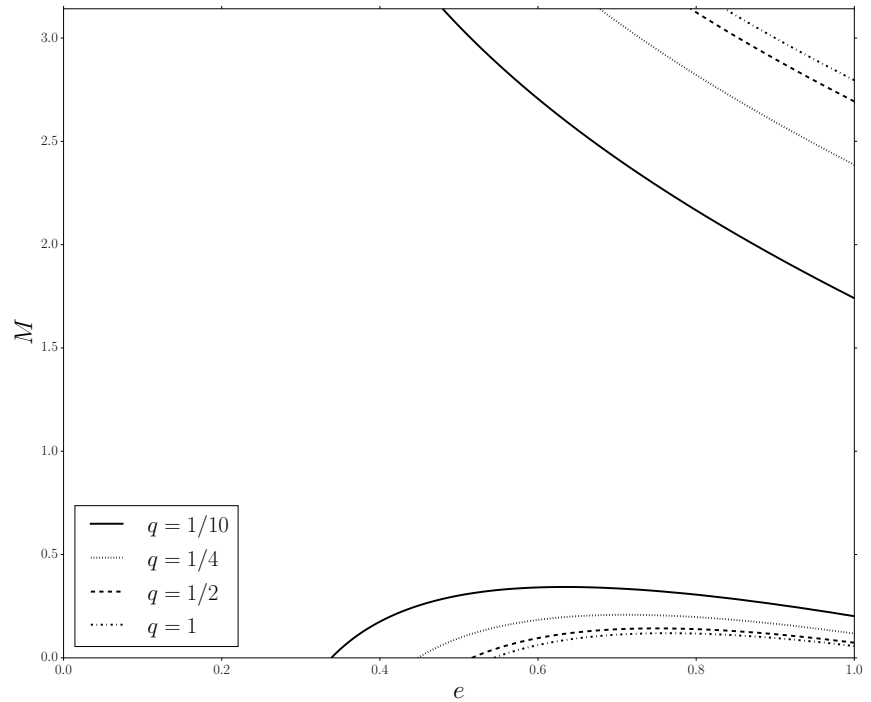

Fig. 8 Boundaries of the $\Sigma_{q}\left(E_{0}\right)$ sets of starter $S_{4}: E_{0}=M+e$ for $q=1,1 / 2,1 / 4,1 / 10$. Here the sets $\Sigma_{q}$ include the points $(e, M) \in[0,1) \times[0, \pi]$ under the upper boundary and above of the lower boundary.

with $M_{0}=0, M_{1}=1-e, M_{2}=\pi-1-e, M_{3}=\pi$ and

$$
\begin{aligned}
& p_{1}=\frac{\eta_{1}}{1-e}, p_{2}=\frac{\eta_{2}}{\pi-2}, \quad p_{3}=\frac{\pi-\eta_{1}-\eta_{2}}{1+e}, \\
& q_{1}=0, \quad q_{2}=\eta_{1}+\frac{(e-1) \eta_{2}}{(\pi-2)}, q_{3}=\frac{\pi\left(\eta_{1}+\eta_{2}\right)+\pi(1-e-\pi)}{1+e},
\end{aligned}
$$

and

$$
\begin{aligned}
& \eta_{1}=\frac{a_{0}+a_{1} e}{1+a_{2} e}, a_{0}=1, \quad a_{1}=-0.633589, a_{2}=-0.564096 \\
& \eta_{2}=\frac{b_{0}+b_{1} e}{1+b_{2} e}, b_{0}=\pi-2, b_{1}=-0.860154, b_{2}=-0.777978 .
\end{aligned}
$$

Next, in Figure 12 we display the $q$-convergence sets for $q=1,1 / 2,1 / 4,1 / 10$.

\subsection{The starter of Markley.}

Markley (1995) proposed a very accurate starter $S_{M}$ with a quite large computational cost that combined with a fifth-order iterative method gives in one iteration a maximum relative error less than $10^{-18}$ when roundoff errors and double precision are properly addressed.

In this starter $E=E_{0}$ is the exact solution of the cubic equation

$$
[3(1-e)+\alpha e] E^{3}-3 M E^{2}+6 \alpha(1-e) E-6 \alpha M=0,
$$




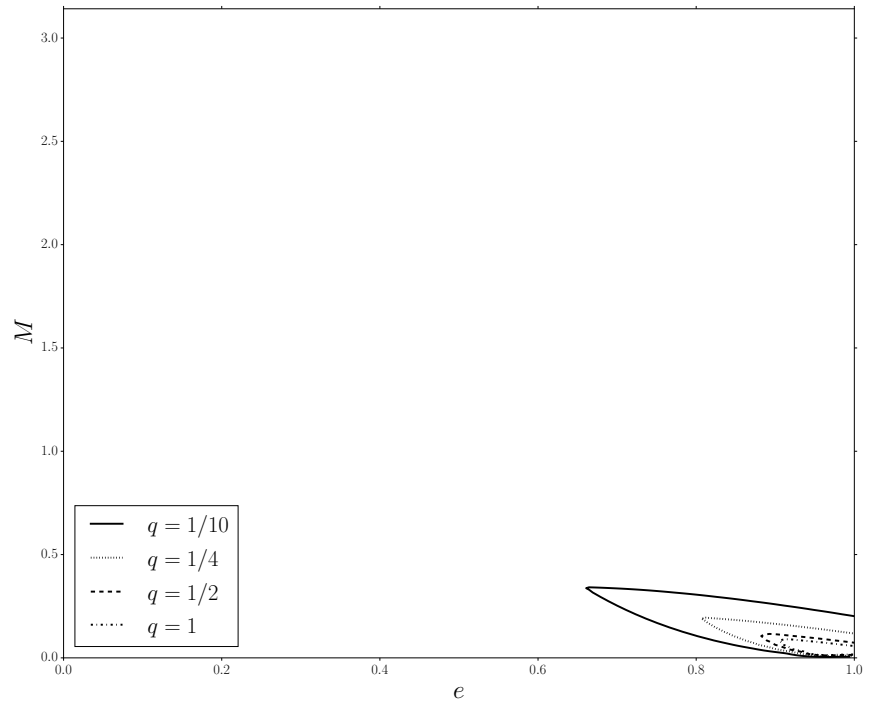

Fig. 9 Boundaries of $\Sigma_{q}\left(E_{0}\right)$ sets of starter $S_{7}$ : $(31)$ for $q=1,1 / 2,1 / 4,1 / 10$. The corresponding sets are limited between the upper and lower boundaries.

where $\alpha$ is, in principle, a specified constant. Here we have chosen the value

$$
\alpha=\frac{3 \pi^{2}}{\left(\pi^{2}-6\right)}
$$

although a non constant improved value of $\alpha$ is

$$
\alpha(e, M)=\frac{3 \pi^{2}+1.6 \pi(\pi-M) /(1+e)}{\pi^{2}-6} .
$$

Note that (34) arises by substituting in KE the function $\sin E$ by the Padé approximation

$$
\frac{E\left(6 \alpha-(\alpha-3) E^{2}\right)}{6 \alpha+3 E^{2}}
$$

From equation (34) it follows that

$$
M=\frac{(3(1-e)+\alpha e) E_{0}^{3}+6 \alpha(1-e) E_{0}}{6 \alpha+3 E_{0}^{2}},
$$

and this implies that in the case of Markley starter the function $\lambda=\lambda\left(S_{M}\right)$ of (16) can be written explicitly as a function of $\left(e, E_{0}\right) \in[0,1) \times[0, \pi]$ instead of the variables $(e, M) \in[0,1) \times[0, \pi]$ and is given by

$$
\lambda\left(S_{M}\right)=\frac{e\left|E_{0}-e \sin E_{0}-M\right|}{\left(1-e \cos E_{0}\right)^{2}}=-e^{2} \frac{-6 \pi^{2} E_{0}+6 E_{0}^{3}+\left(-6 E_{0}^{2}+\pi^{2}\left(6+E_{0}^{2}\right)\right) \sin E_{0}}{\left(-6 E_{0}^{2}+\pi^{2}\left(6+E_{0}^{2}\right)\right)\left(1-e \cos E_{0}\right)^{2}} .
$$




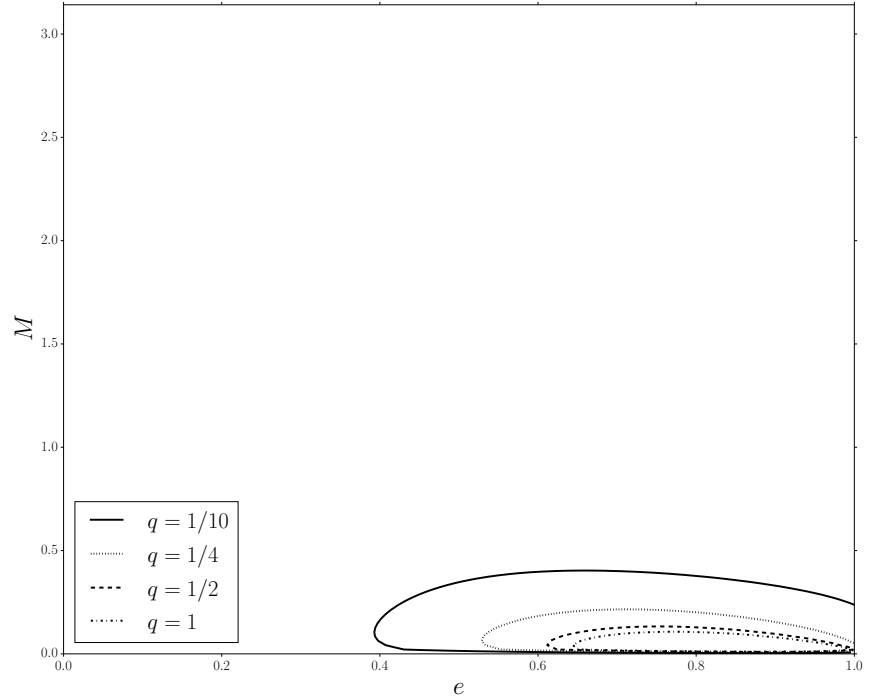

Fig. 10 Boundaries of $\Sigma_{q}\left(E_{0}\right)$ sets of starter (32) for $q=1,1 / 2,1 / 4,1 / 10$. Here the $\Sigma_{q}$ sets includes all points $(e, M) \in[0,1) \times[0, \pi]$ outside the corresponding boundaries.

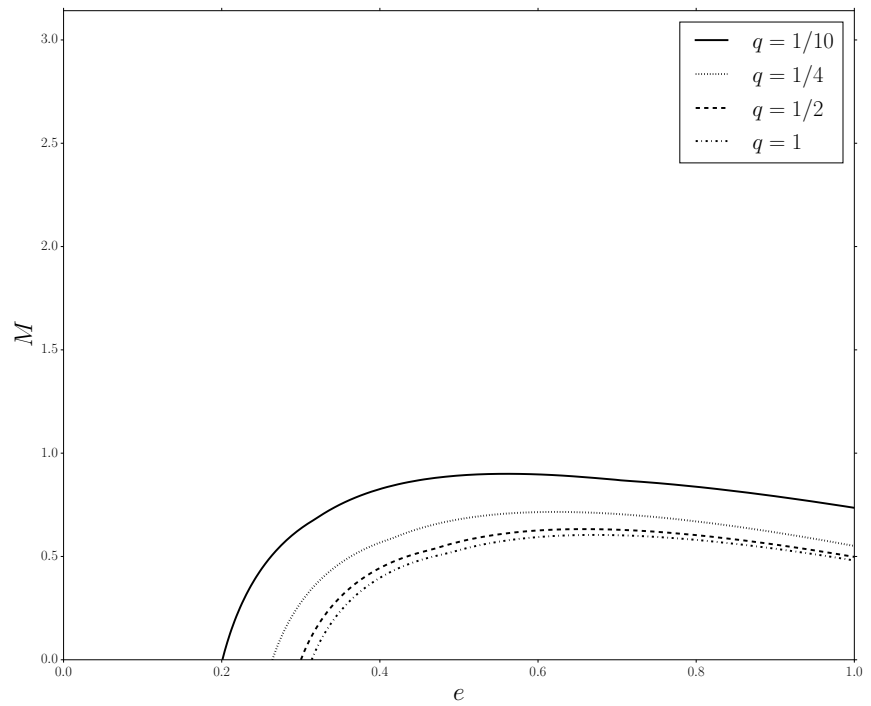

Fig. 11 Boundaries of $\Sigma_{q}\left(E_{0}\right)$ of Broucke's starter for $q=1,1 / 2,1 / 4,1 / 10$. Here the $\Sigma_{q}-$ sets includes all points $(e, M) \in[0,1) \times[0, \pi]$ outside the corresponding boundaries. 


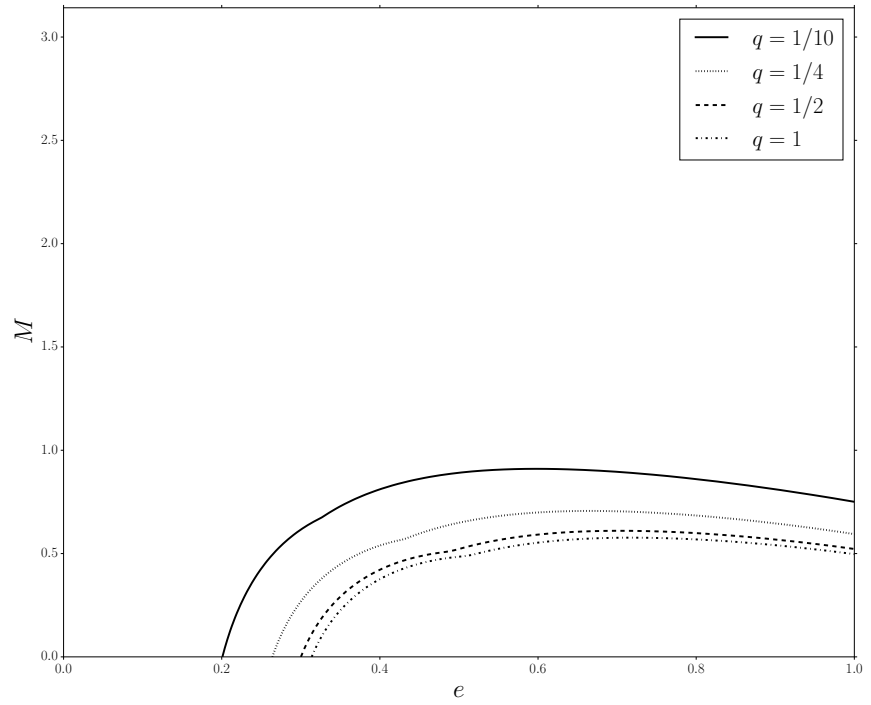

Fig. 12 Boundaries of $\Sigma_{q}\left(E_{0}\right)$ sets of CEMR's starter in the $(e, M)$ plane for $q=$ $1,1 / 2,1 / 4,1 / 10$

Note that $E_{0}(e, \cdot):[0, \pi] \rightarrow[0, \pi]$ is a one to one map. Because of this we will consider $\lambda\left(S_{M}\right)=\lambda\left(S_{M}\right)\left(e, E_{0}\right)$.

By using (37) we may check that $f_{e}\left(E_{0} ; M\right) \geq 0$ for all $e \in[0,1), E_{0} \in[0, \pi]$. Hence $E_{0}(e, M) \geq E^{*}(e, M)$ and Markley starter is a monotonic starter for all $e \in[0,1)$, $E_{0} \in[0, \pi]$.

It can be seen that

$$
\sup \left\{\lambda\left(S_{M}\right) ;\left(e, E_{0}\right) \in[0,1) \times[0, \pi]\right\}=\lambda\left(S_{M}\right)(1,2.20982)=0.0171415=\nu_{M},
$$

and $q\left(\nu_{M}\right)=0.00872089$ therefore this starter is $q$-convergent with a factor $q<q\left(\nu_{M}\right)$ for all $(e, M) \in[0,1) \times[0, \pi]$.

In Figure 13 we display the boundaries of the sets $\Sigma_{q}\left(S_{M}\right)$ for $q=5 / 1000,1 / 1000,1 / 10000$.

Here we have chosen much smaller values of $q$ than in the above starters because in this case the starter is very accurate and Newton's convergence is very fast.

Clearly the above behaviour of the $q$-convergence together with the fact that

$$
\sup \left\{\left|N f_{e}\left(E_{0}\right)-E_{0}\right| ;\left(e, E_{0}\right) \in[0,1) \times[0, \pi]\right\}=0.0136618
$$

implies a very fast convergence for all $(e, M) \in[0,1) \times[0, \pi]$ for this starter. The calculation of scaled $q$-convergence regions and error bounds can be carried out as in the first starter. 


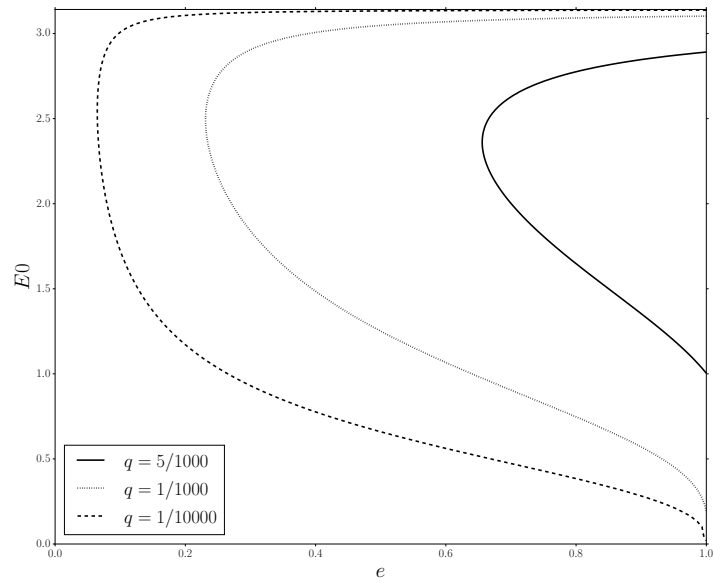

Fig. 13 Boundaries of $\Sigma_{q}\left(E_{0}\right)$ sets $\Sigma_{q}\left(S_{M}\right)$ of Markley's starter for $q=$ $5 / 1000,1 / 1000,1 / 10000$. The corresponding regions are on the left side of the boundaries.

3.11 The starter $S_{10}$.

By substituting in $\mathrm{KE} \sin E$ by their third-order truncated expansion at $E=0$ we have the cubic equation in the eccentric anomaly

$$
P_{e}(E ; M)=(1-e) E+e\left(E^{3} / 6\right)-M=0 .
$$

Now in the starter $S_{10}, E_{0}=E_{0}(e, M)$ is defined as the solution of (39) for $e \in$ $[0,1), M \in[0, \pi]$.

First of all observe that $P_{e}(0 ; M)=-M \leq 0$ and $P_{e}(\pi ; M)>0$ together with the fact that $P_{e}^{\prime}(E, M)=(1-e)+e E^{2} / 2>0$ implies that for all $e \in[0,1), M \in[0, \pi]$ the equation (39) has a unique solution. Further for such a solution $E=E_{0}$ we have

$$
f_{e}\left(E_{0} ; M\right)=e\left[\left(E_{0}-\frac{E_{0}^{3}}{6}\right)-\sin E_{0}\right]<0
$$

from the monotonic increasing and the convexity of $f_{e}$ with respect to $E$ it follows that $E_{0}(e, M)<E^{*}(e, M)$ and the first Newton's iteration $E_{1}=N_{f}\left(E_{0}\right)>E^{*}$. Also it can be seen that $E_{1} \leq \pi$, so the remaining iterations decrease montoniquely to the exact solution $E^{*}(e, M)$.

Taking into account (39) for the $\lambda$-function (16) we get

$$
\lambda\left(S_{10}\right)=-\frac{e^{2}\left(-6 E_{0}+E_{0}^{3}+6 \sin E_{0}\right)}{6\left(1-e \cos E_{0}\right)^{2}},
$$

where $E_{0}=E_{0}(e, M)$ is defined by (39). In Figure 14 we display the boundaries of the $q$-convergence sets $\Sigma_{q}\left(S_{10}\right)$ for $q=1,1 / 2$

A remarkable fact already noted in Avendaño et al. (2014) is that these $q$-convergence regions include a neighborhood of the singular point $(e, M)=(1,0)$ in the Newton's 


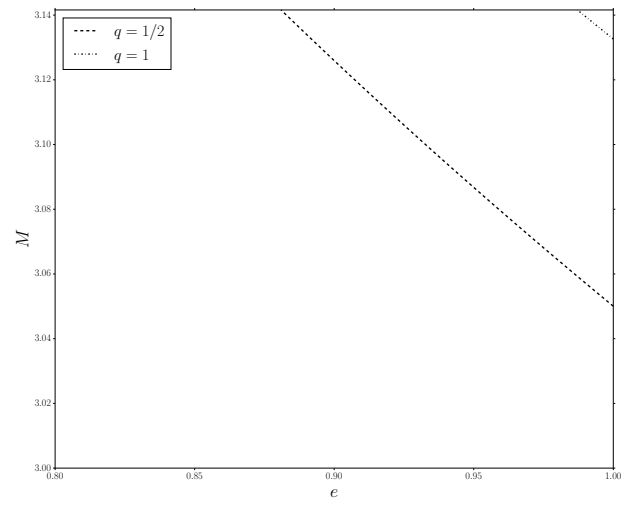

Fig. 14 Boundaries of $\Sigma_{q}\left(E_{0}\right)$ sets (in white) $\Sigma\left(S_{10)}\right.$ around the point $(e, M)=(1, \pi)$

iteration in contrast with some other previous starters. This fact has been used by Avendaño and co-workers Avendaño et al. (2014) to construct a globally simple starter in the whole set $(e, M) \in[0,1) \times[0, \pi]$ that satisfies the $\alpha$-test i.e is $q$-convergent with factor $q=1 / 2$.

Also it must be noticed that in Avendaño et al. (2014) it has been proved that $S_{10}$ satisfies the $\alpha$-test for all $(e, M) \in[0,1) \times[0, \pi]$. However as can be seen in Figure ?? the sufficient condition of $q$-convergence derived from Proposition 1 does not guarantee this property for $q=1 / 2$. In fact

$$
\lambda\left(S_{10}\right)(1, \pi)=\frac{\pi\left(\pi^{2}-6\right)}{24}>\frac{4}{9}
$$

and by continuity the set $\Sigma_{1 / 2}\left(S_{10}\right)$ does not include a neighborhood of $(e, M)=(1, \pi)$. This implies that the sufficient conditions of $q$-convergence of $\alpha$-test and Proposition 1 can be different for the same starter.

\section{Conclusions}

By using a version of the Newton-Kantorovitch theorem on the convergence of Newton's method for the solution of implicit equations adapted to the KE, we show that it is possible to study the $q$-convergence properties of some well known starters for solving KE. In particular for a given starter $S$ we may determine the sets $\Sigma_{q}(S)$ of $(e, M) \in$ $[0,1) \times[0, \pi]$ in which we may ensure $q$-convergence with rate $q<1$. Also we may compute the scaled convergence sets in which the convergence rate has the form $q=$ $e^{k} \widetilde{q}$ for $k \geq 1$ and therefore tends to zero as the eccentricity tends to zero. The theorem allows us to derive for each starter a priori and a posteriori error bounds on the iteration error depending on the number of iterations and $(e, M)$. Thus we present here a number of tools that allow to a practical user to choose the most efficient and accurate starter (or some combination of them) according their particular requirements. 


\section{Acknowledgements}

This work has been supported by the Spanish Ministry of Economy, projects ESP201344217-R, DGI MTM2013-47318-C2-1-P.

\section{References}

Argyros, I.K., Hilout, S., Khattri, S.K.: Expanding the applicability of Newton's method using Smale's $\alpha$-theory J. Comput. App. Math. 261, 183-200 (2014)

Avendaño, M., Martín-Molina, V., Ortigas-Galindo, J.: Solving Kepler's equation via Smale's $\alpha$-theory. Celest. Mech. Dyn. Astron. 119, 27-44 (2014)

Avendaño, M., Martín-Molina, V., Ortigas-Galindo, J.: Approximate solutions of the hyperbolic Kepler equation. Celest. Mech. Dyn. Astron. 123, 435-451 (2015)

Blum, L., Cuker, F., Shub, M. and Smale, S.: Complexitiy and Real Computations.Part II: Some Geometry of Numerical Algorithms, City University of Hong Kong, preprint (2010)

Broucke, R. On Kepler's equation and strange attractors. J. Astronaut. Sci. 28, 255$265(1980)$

Calvo, M., Elipe, A., Montijano, J.I., Rández, L.: Optimal starters for solving the elliptic Kepler's equation. Celest. Mech. Dyn. Astron. 115, 143-160 (2013)

Calvo, M., Elipe, A., Montijano, J.I., Rández, L.: Convergence of starters for solving Kepler's equation via Smale's $\alpha$-test, Celest. Mech. Dyn. Astron. 127, 19-34 (2017)

Charles, E.D. and Tatum, J.B.: The convergence of Newton-Raphson iteration with Kepler's equation, Celest. Mech. Dyn. Astron. 69, 357-372 (1998)

Colwell P.: Solving Kepler's equation over three centuries. Willmann-Bell, Richmond, VA (1993)

Davis J.J., Mortari D., Bruccoleri C.: Sequential solution to Kepler's equation. Celest. Mech. Dyn. Astron. 108, 59-72 (2010)

Dedieu, J.P.: Points Fixes, Zeros et la Mèthode de Newton. Mathematics and Applications 54. Springer. Berlin (2006)

Dubinov, A.E. and Galidakis, I.N.: Explicit solution of the Kepler equation, Phys. Part. Nuclei Lett. 4, 213-216 (2007)

Feinstein, S.A., McLaughlin, C.A.: Dynamic discretization method for solving Kepler's equation. Celest. Mech. Dyn. Astron. 96, 49-62 (2006)

Kantorovich, L.V, G.P. Akilov, G.P.: Functional Analysis. Pergamon Press. Oxford (1982)

Markley, F.L.: Kepler equation solver. Celest. Mech. Dyn. Astron. 63, 101-111 (1995)

Mikkola, S.: A cubic approximation for Kepler's equation. Celest. Mech. 40, 329-334 (1987)

Mortari, D., Clochiatti, A.: Solving Kepler's Equation using Bézier curves. Celest. Mech. Dyn. Astron. 99, 45-57 (2007)

Odell, A.W., Gooding R.H.: Procedures for Solving Kepler's Equation. Celest. Mech. Dyn. Astron. 38, 307-334 (1986)

Ortega J. M., Rheinboldt W. C.: Iterative Solution of Nonlinear Equations in Several Variables. Academic Press, New York, (1970)

Palacios, M.: Kepler equation and accelerated Newton method. J. Comput. App. Math. 138, 335-346 (2002) 
Smale, S.: Newton's Method estimates from Data at One Point. In: The Merging of Disciplines in Pure, Applied and Computational Mathematics. Springer Verlag. New York-Berlin, 185-196 (1986)

Smith, G.R.: A simple Efficient Starting Value for the solution of Kepler's Equation for Elliptic Orbits, Celest. Mech. 19, 163- (1979).

Wang Xinghua: Convergence of Newton's method and inverse function theorem in Banach space. Mathematics of Computation 68, 225, 169-186 (1999) 